\title{
Good and bad news for an antiangiogenic
}

A vastin, a VEGF-specific monoclonal antibody originally designed to inhibit angiogenesis (Figure 1), has recently made the headlines with both positive and negative clinical progress. At the start of May, it was approved by the FDA for use as a treatment for glioblastoma that has recurred after standard therapies, but in April a clinical trial assessing the ability of Avastin to reduce the risk of tumor recurrence in individuals with early-stage colon cancer failed to reach its endpoint.

Until the start of May, Avastin, which is made by Genentech/Roche, was FDAapproved only for the treatment of the following types of cancer: metastatic colorectal cancer; unresectable, locally advanced, recurrent, and metastatic nonsquamous non-small cell lung cancer; and metastatic HER2-negative breast cancer (that has not been treated with chemotherapy).

In addition to these clinical contexts, Avastin is used "off-label" by clinicians to treat a range of diseases, including agerelated macular degeneration, where it reduces visual impairment. Positive effects following off-label use have led to a number of clinical trials, some of which were behind the FDA approval of Avastin for use in treating glioblastoma.

In light of Avastin's recent mixed fortunes in clinical settings, the JCI talked to one scientist who focuses on tumor angiogenesis, Rakesh K. Jain, Professor of Tumor Biology at Massachusetts General Hospital and Harvard Medical School, to

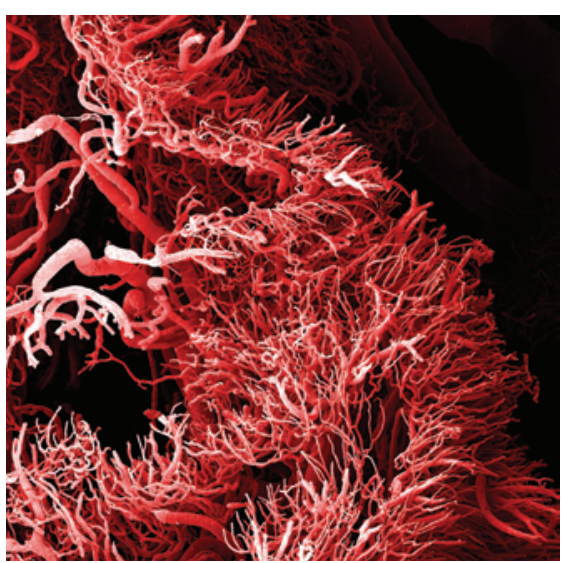

Figure 1

Tumor angiogenesis is required for tumor expansion. Image credit: Clouds Hill Imaging Ltd./Photo Researchers Inc. gain insight into the future of cancer therapeutics designed to inhibit angiogenesis.

JCI: A clinical trial studying the effects of the antiangiogenic drug Avastin in nonmetastatic colon cancer recently failed to achieve its endpoint. Do you think this will dampen enthusiasm for further development of similar drugs?

Jain: No. Antiangiogenic agents show clear activity and promise in many tumors. While the gains in overall survival are modest, a subset of patients benefit significantly. As we learn how to choose these patients, and optimize the dose and schedule, the enthusiasm will increase again significantly.

JCI: One of the founding fathers of antiangiogenic therapy as a treatment for cancer, Judah Folkman, passed away last year and is being honored at a forthcoming NYAS meeting at which you are speaking about the future of this therapeutic approach. Tell us a little more about where you think the field is heading.

Jain: As we better understand the mechanism(s) of action of these drugs and establish biomarkers of benefit and toxicity, we will be able to select patients and rationally design treatment protocols that yield improved outcome. However, we also need to determine the alternative molecular and cellular pathways responsible for resistance to VEGF blockade. These will be likely agentand disease-specific. Moreover, some of these may be common to other processes, such as neuronal guidance. Another emerging direction with many applications is to examine the interaction between angiogenesis, vascular normalization, and the immune system.

JCI: What do you think are the most interesting developments in tumor angiogenesis basic science research in the last 12 months?

Jain: We've seen several exciting results regarding the molecular determinants of vascular normalization in tumors. For example, crucial roles in determining various abnormal characteristics of the tumor vasculature have been ascribed to prolyl hydroxylase domaincontaining protein 2 ( $\mathrm{Phd} 2$ ) in endothelial cells (1), regulator of G-protein signalling 5 (Rgs5) in pericytes (2), and VEGF-A in myeloid cells (3). Other data indicate that PDGF-C might have a role in attenuating the antiangiogenic effects of VEGF- and VEGFR2-specific monoclonal antibodies (4, 5). Finally, Greenberg et al. have identified a role for VEGF, acting via a receptor complex consisting of PDGF-R $\beta$ and VEGFR2, as a negative regulator of pericyte function and vascular normalization (6). All these basic discoveries provide novel ways to normalize abnormal vessels and thereby develop new anticancer therapeutics.

JCI: On a more personal note, you have just been elected to the NAS. How did you feel when you found out, and what do you consider your biggest scientific accomplishment?

Jain: I was pleased and honored to be elected to the NAS, and grateful to my mentors, students, collaborators, coworkers, and colleagues, as well as my family, for their unyielding support. I think that my biggest scientific accomplishments are putting forward the hypothesis that antiangiogenic therapy could "normalize" the abnormal tumor vasculature and microenvironment, and thus improve both the delivery and efficacy of conventional and novel therapies, and providing preclinical and clinical evidence to support the hypothesis (7). Vascular normalization has now been validated by a number of laboratories worldwide and has changed the thinking about how antiangiogenic agents work in patients. For example, we have shown that a vascular normalization index (VNI) correlates with survival in glioblastoma patients receiving a pan-VEGFR tyrosine kinase inhibitor. If validated in a Phase III trial, the VNI has the potential to become a biomarker for selecting patients who are most likely to benefit from anti-VEGF therapies.

\section{Karen Honey}

1. Mazzone, M., et al. 2009. Heterozygous deficiency of PHD2 restores tumor oxygenation and inhibits metastasis via endothelial normalization. Cell. 136:839-851.

2. Hamzah, J., et al. 2008. Vascular normalization in Rgs5-deficient tumours promotes immune destruction. Nature. 453:410-414.

3. Stockmann, C., et al. 2008. Deletion of vascular endothelial growth factor in myeloid cells accelerates tumorigenesis. Nature. 456:814-818.

4. Crawford, Y., et al. 2009. PDGF-C mediates the angiogenic and tumorigenic properties of fibroblasts associated with tumors refractory to antiVEGF treatment. Cancer Cell. 15:21-34.

5. di Tomaso, E., et al. 2009. PDGF-C induces maturation of blood vessels in a model of glioblastoma and attenuates the response to anti-VEGF treatment. PloS ONE. 4:e5123.

6. Greenberg, J.I., et al. 2008. A role for VEGF as a negative regulator of pericyte function and vessel maturation. Nature. 456:809-813.

7. Jain, R.K. 2001. Normalizing tumor vasculature with anti-angiogenic therapy: a new paradigm for combination therapy. Nat. Med. 7:987-989. 